Verordnung des Beauftragten für den Vierjahresplan über die Behandlung der Erfindungen von Gefolgschaftsmitgliedern gesetzlich verankert worden (Reichsgesetzblatt I S. 466). Darin wird verfügt, daB in den privaten und öffentlichen Betrieben unter Aufsicht der Gauämter für Technik der NSDAP für eine geeignete Betreuung der erfinderisch tätigen Gefolgschaftsmitglieder $z \mathfrak{u}$ sorgen ist. Die Verordnung verpflichtet ferner jedes Gefolgschaftsmitglied die von ihm gemachte Erfindung, soweit sie aus seiner Arbeit im Betrieb heraus entstanden ist, dem Unternehmer zur Verfügung zu stellen. Der Unternehmer hat dafür eine angemessene Vergütung zu zahlen.

Im Gegensatz zum Patentgesetz vom 5. Mai 1936, das nur die i de ell e A ne rke nn ung der Erfinder-Ehre sicherte, bringt die neue Verordnung nun auch den gesetzlichen Anspruch des Gefolgschaftsmitgliedes auf eine angemessene Vergütung für die Erfindung, die von seinem Unternehmer in Anspruch genommen wird. Damit wird eine vom Hauptamt für Technik der NSDAP (Reichsleitung) seit Jahren vertretene Forderung zur Sicherung der schöpferischen Leistungskraft des deutschen Volkes verwirklicht.

\title{
Reise der Internationalen Forstzentrale in die Slowakei
}

Auf Einladung der slowakischen Regierung unternahmen die ständigen Mitarbeiter der Internationalen Forstzentrale unter der Führung von Professor Dr. Dr. KöSTLER in der Zeit vom 6.-I3. Juli 1942 eine Informationsreise durch slowakische Waldgebiete, die im Zusammenhang mit den Aufgaben der Internationalen Forstzentrale insbesondere der Unterrichtung über die Verhältnisse des slowakischen Forstwesens und der holzwirtschaftlichen Industrie des Landes diente. Am Tage der Ankunft in PreBburg hielt Sektionsrat BEDNARCIK, Delegierter der Slowakei beim Komitee des. C. I. S., einen einführenden Vortrag über die Verhältnisse der slowakischen Forst- und Holzwirtschaft, der bei den folgenden Waldbesichtigungen durch weitere, örtliche Ausführungen der Herrn Obersektionsrat KLUCAR und Ministerialrat BLATtNy sowie der Außenbeamten vervollständigt wurde. Dank des sorgfältig zusammengestellten Bereisungsplanes sowie der ausgezeichneten Führung durch die genannten Herren und ihrer erschöpfenden Erläuterungen konnte reichhaltiges Studienmaterial für die fachliche Arbeit der Internationalen Forstzentrale gesammelt werden. Die Reise, an der zeitweilig auch der Forstattaché an der Deutschen Gesandtschaft in Prebburg, Oberforstmeister Dr. Wechselberger, teilnehmen konnte, begann in der Westslowakei und führte durch sämtliche Staatsforstdirektionen bis in das Gebiet der Hohen Tatra. Außer den Staatswaldungen wurden auch Forste der Militärverwaltung sowie Kommunalwälder, Urbarialwaldungen und einige nach modernsten Gesichtspunkten eingerichtete staatliche Sägewerke besichtigt.

Durch die auf dieser Informationsreise gewonnenen unmittelbaren Einblicke in die Forst- und Holzwirtschaft eines der waldreichsten Staaten Europas konnten die Mitarbeiter der Internationalen Forstzentrale die für ihre Arbeit wichtige Kenntnis der forstlichen Verhältnisse einzelner Länder wesentlich erweitern. Ein eingehender Reisebericht wird in der Zeitschrift Intersylva Nr. 4, 1942, erscheinen.

\section{Sei sparsam im Verbrauch von Strom und Gas!}

und Du hilfst Waffen schmieden, trägst dazu bei, daß Räder rollen im Dienst der Front und der Heimat, hilfst den Sieg erringen und damit die Zukunft unseres Volkes sichern. Brenne nicht unnötig Glühlampen, schalte Dein Rundfunkgerät aus, wenn $\mathrm{Du}$ es nicht wirklich brauchst, gehe sparsam um mit elektrischen und Gasgeräten, denke daran, daß es auf Jeden ankommt, viele Wenig machen ein Viel. Sparsamkeit mit Strom und Gas bedeutet Sparsamkeit im Verbrauch von Kohle, die der Bergmann in harter Arbeit dem Schoße der Erde abringen muß. 